\title{
Relation between Mammographic Parenchymal Patterns and Breast Cancer Risk: Considering BMI, Compressed Breast Thickness and Age of Women in Tabriz, Iran
}

\author{
Parinaz Mehnati ${ }^{1 *}$, Hamed Alizadeh ${ }^{1}$, Haleh Hoda ${ }^{2}$
}

\begin{abstract}
Background: Mammographic density determined according paranchymal patterns is a risk factor for breast cancer and its relationships with body and other breast characteristics of women is important. The purpose of the present study was to correlate breast parenchymal patterns and mammography abnormality findings with women's BMI, compressed breast thickness (CBT) and age in Tabriz city, Iran. Materials and Methods: From 1,100 mammograms interpreted by radiologists, breast parenchymal was classified into four categories from Types 1 (mostly fatty) through 4 (mostly fibroglandular tissue). Age, BMI, and CBT were recorded and their relation with risk for the development of breast abnormalities in mammograms was analyzed. Results: In women with a mean age of $45.8 \pm 8.63$ years $17.7 \%$ were in the high density group (Type 3 and 4 ). A comparison of four types of breast paranchymal with BMI, CBT and age showed inverse relations to breast density. Abnormal mammographic findings were $25.8 \%$ of all reported mammograms with a circular mass $(\mathbf{1 2 . 7 \%})$ as the most common abnormality. About $21 \%$ abnormal cases were in less than 40 years. Increasing of BMI had significant relation with breast abnormality but in CBT was not observed. Conclusions: Measurement of women's body characteristics is useful for assistance in mammography diagnosis as well as selection of imaging instrument by high sensitivity for following patient in future. The effects of age, CBT and BMI groups on the breast paranchymal were significant.
\end{abstract}

Keywords: Breast parenchymal - abnormality - age - BMI - CBT

Asian Pac J Cancer Prev, 17 (4), 2259-2263

\section{Introduction}

Mammography is a well-accepted diagnostic technique for the early detection of breast cancer and has been shown to reduce the breast cancer mortality rate in women who are 40-69 years old Boonlikit (2013). Some studies explained age and race related changes in mammographic parenchymal patterns (Zulfiqar et al., 2011). The radiology appearance of the female breast varies from completely fatty to extremely dense, depending on the proportion of fibroglandular tissue present.

It has been demonstrated that the mammographic parenchymal pattern or the mammographic density of the breast is related to the risk of developing breast cancer (Ciatto and Zappa 1993; Sickles, 2007) with a two-six fold increase in risk in women with a dense parenchyma pattern compared with women with a fatty paranchymal pattern. In young women, breast parenchymal pattern is more commonly dense. The breast density naturally diminishes with age. But hormone replacement therapy slows breast involution and increases mammographic density (Ahmadi et al., 2005). Also, Denser breast is seen in nulliparous women and women with later first pregnancies (age $>20$ years) than multiparous women (Mehnati and Tirtash Jafari, 2015).

From 1976 until 1980 the articles demonstrated a doubtful relationship between women breast characteristic and breast cancer risk. But since 1982, many studies in which quantitative methods were used for assessing breast density; showed a positive moderate association with breast cancer risk (Kato et al., 1995; Hosseinzadeh et al., 2014). A study showed that breast density and age significantly affect the accuracy of mammography diagnosis (Boyd et al., 2007).

Breast cancer is the most common cancer in Asian women and the second cause of death from cancer in women. The incidence of breast cancer in Asian countries is lower but cause to specific-mortality is much higher when compared with western countries (Youlden et al., 2014). The incidence of breast cancer in Iran was 22 per 100,000 but changed to 28 per 100,000 from 2005 to 2009, respectively (Harirchi et al., 2004; Taheri et al., 2012). The highest frequency of malignancies was observed in the $40-49$ age groups $(31.8 \%)$. Twenty-three percent of 
breast cancers were observed in women younger than 40 years. About 83 percent of malignant lesions in women were in $\mathrm{T} 2$ and more at diagnosis time. Unfortunately, screening mammography is not defined in Iranian health care system and only academic research projects or women health researches perform sometimes breast screening, with education physical or mammography methods, also, there are some reports that patients consult medical advice and are diagnosed in an advanced stage of breast cancer (Harirchi et al., 2004). Abnormalities seen on mammography in dense breast tissue increase recall rates, reduce test specificity, and may jeopardize the benefit of mammographic diagnostic requests (Mehnati and Jafari Tirtash 2015). Mammographic accuracy was lowest in younger women with dense breasts. Breast density is affected by younger age, weight, body mass index (BMI) and familial or genetic tendencies (Mandelson, et al., 2000).

Most studies suggest that obesity and higher adult BMI are associated with an elevated risk of postmenopausal breast cancer, presumably due to increased estrogen levels produced by excess adipose tissue (Munsell et al., 2014). Although, greater BMI in later adolescence (age 15-18 years) has been inversely associated with both premenopausal and postmenopausal breast cancer risk.

We did not find article which present paranchymal and abnormal mammograms which have been accompanied with age, BMI and CBT studies from Iranian women. The purpose of this study was to describe women's body characteristics including breast parenchymal pattern, age, weight, BMI and breast density among Iranian women and its usefulness for mammography procedure and image diagnosis of breast benign and malignant diseases. Also, It will be a good trick to following of women with different parenchymal according to how is mammography's sensitivity for them.

\section{Materials and Methods}

The patient population for this study is selected from the women with breast problems were visited by a physician and referred to radiology center with mammography request in Tabriz city in Iran. For each patient four mammograms were provided for both breasts as it is routine in craniocaudal (CC) and mediolateral oblique (MLO) views.

The mammograms performed by full-field digital mammography unit (Giotto, Bologna- Italy) almost two years in use. The analysis of the mammographic parenchymal pattern and abnormality finding were performed by two expert radiologists who had more than 10 years experience in mammogram interpret. The mammographic parenchymal patterns assess using Breast Imaging Reporting and Data System (BIRADS) classification (ACR, 2003). The breast parenchyma according to this classification includes Type 1: fatty breast; Type 2: a fatty breast with scattered fibroglandular densities; Type 3: a heterogeneously fibroglandular breast; and Type 4: extremely fibroglandular parenchyma. The densities of the breast on mammograms were divided into two groups: Low (Type 1 and Type 2) and high (Type 3 and
Type 4) breast densities. In order to gather data including age, weight and BMI (weight/hight2), compressed breast thickness (CBT) and paranchymal of women a researcher-made questionnaire form is used. Chosen body characteristics were filled by mammographer careful to patient ethical points. Data analyzed by descriptive statistics ANOV, Whitney U test and Spearman's correlation coefficient.

Recorded age, CBT and BMI of women are classified to before and after 40 years old sub groups for presenting their relation by breast parenchymal and abnormalities. The radiologists' interpretation of parenchymal breast type, existence of an abnormality and type of abnormality are also explained.

\section{Results}

The women with accomplished mammography request were 26-76 years old with mean age of $45.8 \pm 8.63$. There were $80(29.2 \%)$ women below the age of $40,134(48.7 \%)$ who were 41-50 years old, 50 (28.3\%) who were 51-60 years old and $11(3.9 \%)$ women older than 60 years. The CBT between 4 and 5.5 was $38.6 \%$ which was higher frequency of CBT. Mean compressed breast thickness for CC and MLO images recorded 4.9 \pm 1 and $5.8 \pm 1.2 \mathrm{~cm}$, respectively. The most frequent was $5 \mathrm{~cm}$ for both views of breast. The BMI recorded results showed that women with overweight and obese were $35 \%$ of all partnership women. The frequency of each age, weight, BMI and

Table 1. Characteristics of the Studied Women

\begin{tabular}{|c|c|c|}
\hline Body characteristics & Number & Percents \\
\hline \multicolumn{3}{|l|}{ Age (yr) } \\
\hline $26-30$ & 7 & 2.6 \\
\hline $31-35$ & 31 & 11.3 \\
\hline $36-40$ & 42 & 15.2 \\
\hline $41-45$ & 88 & 32 \\
\hline $46-50$ & 46 & 16.7 \\
\hline $51-55$ & 38 & 14 \\
\hline $56-60$ & 12 & 4.3 \\
\hline $61-65$ & 7 & 2.5 \\
\hline $66-70$ & 2 & 0.7 \\
\hline older than 70 & 2 & 0.7 \\
\hline \multicolumn{3}{|l|}{ Weight $(\mathrm{Kg})$} \\
\hline 40.49 & 2 & 0.7 \\
\hline 50.59 & 22 & 8 \\
\hline 60.69 & 90 & 32.7 \\
\hline 70.79 & 81 & 29.5 \\
\hline 80.89 & 48 & 17.4 \\
\hline 90.99 & 17 & 6.18 \\
\hline 100.109 & 4 & 1.45 \\
\hline 110.12 & 1 & 0.3 \\
\hline \multicolumn{3}{|l|}{ BMI } \\
\hline less than 18.5 (Underweight) & 156 & 56.7 \\
\hline $18.5<\mathrm{BMI}<25$ (Normal) & 23 & 8.3 \\
\hline $25<\mathrm{BMI}<30$ (overweight) & 44 & 16 \\
\hline more than 30 (obese) & 52 & 19 \\
\hline \multicolumn{3}{|l|}{$\mathrm{CBT}(\mathrm{cm})$} \\
\hline$<4$ & 86 & 31.1 \\
\hline $4<\mathrm{CBT}<5.5$ & 106 & 38.6 \\
\hline $5.5<\mathrm{CBT}<7$ & 82 & 30 \\
\hline$>7$ & 1 & 0.3 \\
\hline
\end{tabular}


Mammographic Parenchymal Patterns and Breast Cancer Risk: Considering BMI, CBT and Age of Women in Tabriz, Iran Table 2. Mean age, weight, BMI and Thickness in different breast parenchyma types of women

\begin{tabular}{lcccccccc}
\hline & \multicolumn{2}{c}{ Age } & \multicolumn{2}{c}{ weight } & \multicolumn{2}{c}{ BMI } & \multicolumn{2}{c}{ Thickness } \\
\hline & Mean & Std. Deviation & Mean & Std. Deviation & Mean & Std. Deviation & Mean & Std. Deviation \\
\hline Type1 & 47.86 & 9.16 & 74.96 & 14.23 & 30.93 & 5.92 & 5.11 & 1.13 \\
Type2 & 46.05 & 8.40 & 73.70 & 11.97 & 29.80 & 5.02 & 5.00 & 1.08 \\
Type3 & 42.88 & 7.62 & 69.03 & 13.46 & 27.22 & 5.13 & 4.88 & 0.99 \\
Type4 & 40.81 & 6.97 & 66.31 & 10.59 & 23.51 & 2.11 & 4.38 & 1.50 \\
Total & 45.86 & 8.63 & 73.05 & 12.89 & 29.52 & 5.44 & 4.98 & 1.12 \\
\hline
\end{tabular}

Table 3. Relation between Breast Abnormality Existence and Different BMI and Age Groups of Women

\begin{tabular}{lccccc}
\hline BMI.groups & \multicolumn{6}{c}{ Abnormality existence } \\
\hline \multirow{2}{*}{18.5 (Underweight) } & no & yes & no & yes & Age group \\
& 104 & 43 & 5 & 2 & $26-30$ \\
$18.5<$ BMI<25 (Normal) & 20 & 6 & 28 & 14 & $31-35$ \\
& & & 74 & 14 & $41-45$ \\
$25<$ BMI<30 (overweight) & 33 & 13 & 28 & 18 & $46-50$ \\
& & & 30 & 8 & $51-55$ \\
more than 30 (obese) & 47 & 9 & 6 & 6 & $56-60$ \\
& & & 5 & 2 & $61-65$ \\
& & & 1 & 1 & $66-70$ \\
& & & 2 & 0 & older than 70 \\
\hline Total & 204 & 71 & 204 & 71 &
\end{tabular}

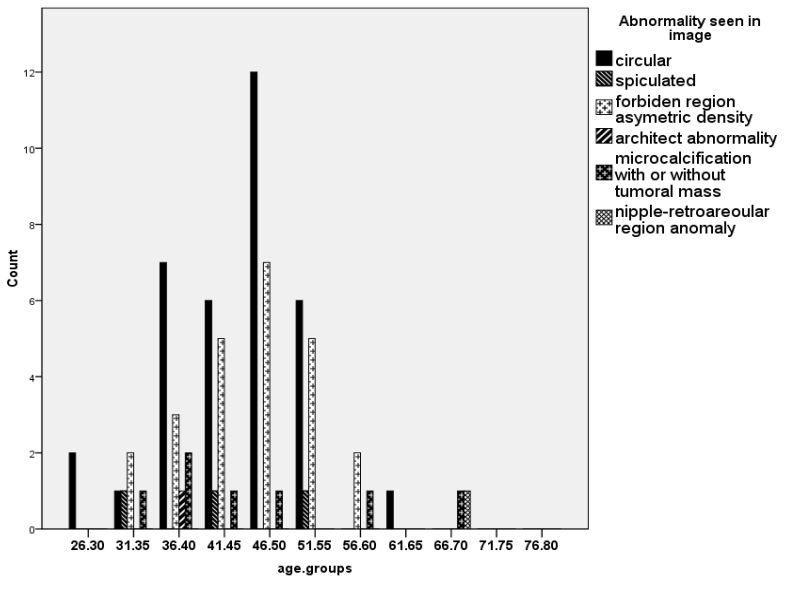

Figure 1. Frequency of Breast Abnormalities by Mammography According to Age Groups

CBT groups of women were explained in detail in Table 1 .

From 1,100 mammograms interpreted by radiologist the majority (54.9\%) of breast parenchymal was Type 2 , $27.4 \%$ of Type $1,11.9 \%$ of Type 3 and $5.8 \%$ Type 4 . Type 3 and 4 (high density group) of breast parenchymals were reduced by increasing of the age and were predominant at the 40 and below. The ratio of high density breasts were $17.7 \%$ in mammograms.

The mean weight, height, BMI and CBT of studied women were $72.7 \pm 1.2 \mathrm{Kg}, 156 \pm 6 \mathrm{~cm}$ and $29.5 \pm 5.4$ and $4.98 \pm 1.12$ as was showed in Table 2. A comparison of four types of breast paranchymal with BMI, CBT and age alterations showed inversely relation to breast density. The significant effects of age, weight and BMI groups on the breast paranchymal pattern were revealed $(\mathrm{p}=0.002$, $\mathrm{p}=0.029$ and $\mathrm{p}=0.016$, respectively).

Abnormal mammographic findings were observed

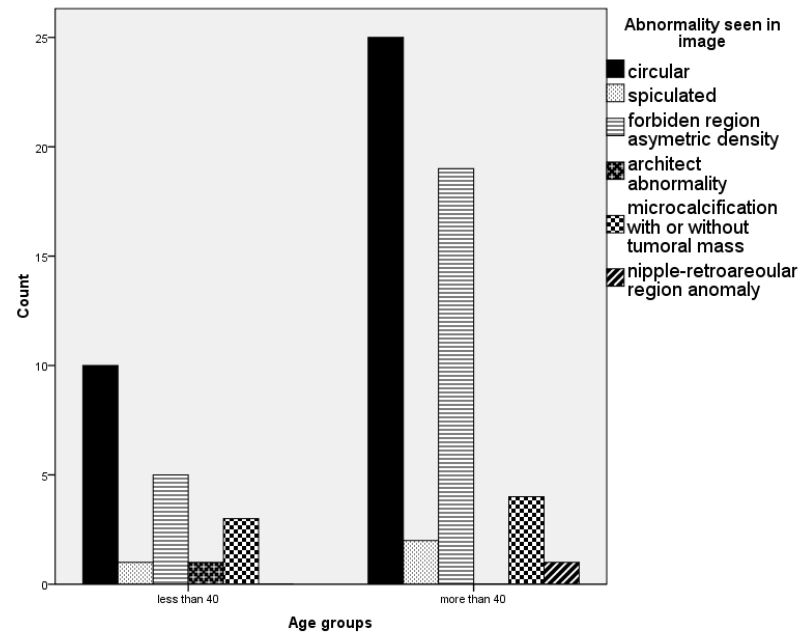

Figure 2. Frequency of Breast Abnormalities of Different Types in Women Older and Younger than 40 Years

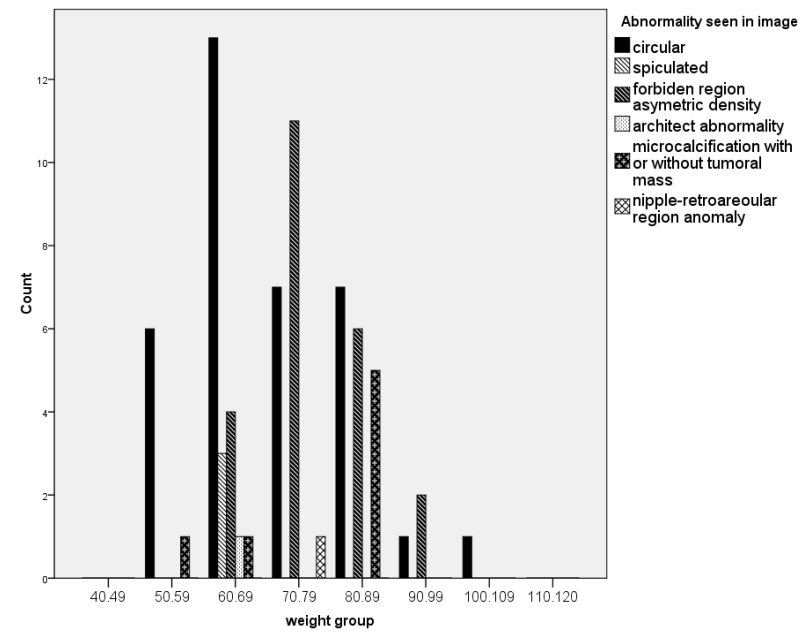

Figure 3. Frequency of Breast Abnormalities by Mammography According to Weight Groups

at about $25.8 \%$ of all reported mammograms (Table 3 ). The frequency of breast abnormalities were $12.7 \%$ circular mass, $8.7 \%$ asymmetric density and $2.5 \%$ micro calcification but speculated mass, breast architect distortion and nipple anomaly have been noted $1.0 \%$ at totality of abnormal cases. A comparison of abnormal mammographic findings and women age groups showed that the high risk groups is $36-55$ years with the $46-50 \mathrm{y}$ (17 cases) the most risky one followed by $36-40 \mathrm{y}$ and 41-45y (13 cases in both). Circular mass was the most common abnormality seen until 60 years old. After 60 years nipple-retroareolar region anomaly becomes the prominent feature (Figure 1).

The women with abnormal finding were classified 
Table 4. Correlation between Abnormal Breast in Women Less and More 40 years and BMI

\begin{tabular}{lccccc}
\hline Age groups & \multicolumn{5}{c}{ BMI groups } \\
\cline { 2 - 6 } & Less than 18.5 & $18.5<$ BMI $<25$ & $25<$ BMI $<30$ & mor than 30 & Total \\
\hline Less than 40 & 51 & 11 & 14 & 14 & 90 \\
More than 40 & 114 & 20 & 39 & 47 & 220 \\
\hline Total & 165 & 31 & 53 & 61 & 310 \\
\hline
\end{tabular}

into two sub groups; less and more than 40 years old for presenting breast abnormality distribution in two women groups. Twenty one abnormal cases were found in under than 40y group as shown in Figure 2. The frequency of weight groups started from 40-49 kg with 6 abnormal cases, $60-69 \mathrm{~kg}$ with 22 and ended by $100-109 \mathrm{~kg}$ by only one case. Circular lesion is observed commonly in each weight sub groups. Also, abnormality types were changing by BMI variation of women (Figure 3 ). A significant direct relation find between increasing of BMI and breast abnormality in women by aging than 40 (Table 4). A CBT interval $4.5-6.5 \mathrm{~cm}$ appeared in more breast abnormalities but in CBT more than $7 \mathrm{~cm}$, abnormality did not find. Data presented that increasing of CBT is not accompany with increasing of breast abnormality.

\section{Discussion}

According to the Iranian Ministry of health and Medical Education in 2004, 7000 women are affected by breast cancer annually and there are 70000 breast cancer patients in this country with a prevalence of $15.5 \%$ IMHME (2004). Unfortunately, in 2007 epidemiological review of breast cancer in Iran has been showed an increasing of incidence ratio of breast cancer in women to 22 per 100000 and in 2010,23\% of all cancers diagnosed in women were breast cancer cases. Also, data presented breast cancer affects Iranian women at least one decade younger than their counterparts in developed countries (40-49 years versus 50-60 years). Thus each procedure which could help to early recognize will be promising.

Why breast paranchymal determination is beneficial? A) Paranchymal pattern classification is basic of determination of breast density as an indicator of breast cancer risk. B) There is a significant relation between increasing of breast density and mammography sensitivity decline in detection of breast cancer because of conceal of some tumors by dense fibroglandular tissue. C) One of the practical usefulness of paranchmal determining is for next screening of women with dense breast that non mammographic imaging such as sonography, optical mammography and MRI is preferred to mammography.

Our study showed a good compatibility of women body characteristics with mammographic findings. We found high risk age groups at 36-55 years old. There is $9 \%$ of breast cancer, in women under 35 years old (Farrokh et all, 2008). In our patients, 9.8\% of abnormal mammograms occurred in younger than 35 , too. Although, a study at 2000-2003 in Tabriz showed malignancy occurred in patient between 50-59 years age (Shakouri Partovi and Nami, 2004) but it seems women breast malignancy is going towards younger age than before in Iran (Mousavi et al., 2007). This should be carefully concern useful information production and any assistance procedure for diagnosis and treatment of breast cancer in younger women.

The mammographic diagnostic sensitivity varied significantly with age and breast density and its accuracy was lowest in younger women with dense breasts. Efficiency of mammography is dependent on its sensitivity and specificity for individual women with different breast density (Mehnati and Jafari Tirtash, 2015). The sensitivity and specificity of mammography according to breast density were $52.1 \%$ and $73.9 \%$, respectively (Devolli-Disha., 2009) but other showed mammographic sensitivity $80 \%$ among women with predominantly fatty breasts but just $30 \%$ in women with extremely dense breasts (Mandelson et al., 2000).

The breasts continue to become less dense, with about $50 \%$ of women in their 40 s and about $65 \%$ of women in their 20s (Boyd et al., 2007). They showed that mammographic appearance of the breasts becomes increasingly radiolucent with the reduction of estrogen and progesterone levels after menopause.

Our findings expressed mean age of 40.81 for high density breast and 47.86 years for low density breast. A shift towards less density paranchymal patterns with aging has been shown in Table 2 .

Although the prevailing literature indicates that breast cancer in women under 40 and especially under 30 is uncommon (Scheibel and Bucciatelli, 2003; Shannon and Smith, 2003) but in our study $29 \%$ of breast abnormalities were observed in women younger than 40 years (Figure 2) and circular mass was the most frequent breast abnormality observed in all age and weight groups before 60 years old. Thus, in a country such as Iran with a predilection of breast cancer for young women, simple observation, which is usually recommended for most of breast caser screening in young woman, is not safe enough. Also, another study in 2004 reported that $23 \%$ of breast cancer determinate in under 40 years women in Iran (Harirchi et al., 2004).

Recorded mean body characteristics compared with the standard women body characteristics recommended by Word Health Organization (http://apps.who.int/bmi/ index.jsp) it showed that weight and BMI were more and height was less than expressed as standard data for women.

Relationship between breast cancer and BMI was significant only in women more than 40 years that it was in agreement with data (Danny et al., 2014) which demonstrated obesity and higher adult BMI is associated with elevated risk of postmenopausal breast cancer. The high risk of abnormality observed in women with Type 2 breast parenchymal pattern but lesser abnormality cases with Type 4 . 
Mammographic Parenchymal Patterns and Breast Cancer Risk: Considering BMI, CBT and Age of Women in Tabriz, Iran

CBT was not noticed in previous researches but this data presented that an increasing of CBT is accompanied by decreasing of breast density, it means small CBT is observed in dense breast, therefore initial prediction of breast parenchymal is possible by CBT measurement.

In conclusion measurement of women's body characteristics is useful for assistance in mammography diagnosis as well as selection of imaging instrument by high sensitivity for following patient in future. The lifetime cancer risk for paranchymal pattern categories among women in different interval of age group was indicated that $29 \%$ of breast abnormalities were observed in women younger than 40 years. A significant direct relation find between BMI and breast abnormality in women by aging than 40 years.

This data presented that small CBT is observed in dense breast, it means prediction of breast parenchymal is possible by CBT measurement even before mammography but increasing of CBT is not accompany with breast abnormality increase.

The effects of age, CBT and BMI groups on the breast paranchymal were significant. In age classified groups near to half of breast abnormal cases were in less than 40 years women. This suggestion of screening program for breast cancer in young and median aged women in Iran urgently needs to start as a national project.

\section{Acknowledgements}

The authors would like to thank the crew of the Golbad Private Radiology clinic, Tabriz, Iran specially Mrs. Zahra Khoshfetrat (radiology technologist) for her assistance in collecting questionnaire form of the research. The current study was supported by the Vice Chancellor for Research (VCR) of Tabriz University of Medical Sciences (TUOMS).

\section{References}

American college of Radiology (2003). Breast Imaging Reporting and Data system (BI-RADS), $4^{\text {th }}$ ed. Reston, VA: American college of Radiology.

Boonlikit S (2013). Comparison of mammography in combination with breast ultrasonography versus mammography alone for breast cancer screening in asymptomatic women. Asian Pac J Cancer Prev, 14, 7731-36.

Boyd NF, Guo H, Martin LJ, et al (2007). Mammographic Density and the risk and detection of breast cancer. $N$ Engl J Med, 356, 227-36.

Ciatto S, Zappa M (1993). A prospective study of the value of the mammographic patterns as indicators of breast cancer risk in a screening experience. Eur J Radiol, 17, 122-25.

Youlden DR, Cramb SM, Yip CH et al (2014). Incidence and mortality of female breast cancer in the Asia-Pacific region. Cancer Biol Med, 11, 101-15.

Devolli-Disha E, Manxhuka-kerliu S, Ymeri H, et al (2009). Comparative accuracy of mammography and ultrasound in women with breast symptoms according to age and breast density. Bosnian J B Med.Scie, 9, 131-6.

Farrokh D, Zandi B, Hashemi J (2008). Study of mammographic findings of breast cancer in women under 35 years old. Iranian $J$ Radiol, 5, 55.

Harirchi I, Karbakhsh M, Kashefi A (2004). Breast cancer in
Iran: results of a multi-center study. Asian Pac J Cancer Prev, 5, 24-7.

Hosseinzadeh M, Eivazi Ziaei J, Mahdavi N et al (2014). Risk factors for breast cancer in Iranian women: a hospital-based case- control study in Tabriz, Iran. J Breast Cancer, 17, $236-43$

Iran Ministry of Health and Medical Education (2004). Disease management center, cancer office. Country report of cancer cases. Tehran: kelk Zarrin press, p.16 (in Persian).

Kato I, Beinart C, Bleich A, et al (1995). A nested case-control study of mammographic patterns, breast volume, and breast cancer. Cancer Couse Control, 6, 431-38.

Mandelson MT, Oestreicher N, Porter PL, et al (2000). Breast density as a predictor of mammographic detection: comparison of interval- and screen-detected cancers. J Natl Cancer Inst, 92, 1081-87.

Mehnati P,Tirtash Jafari M (2015). Comparative efficacy of four imaging instruments for breast cancer screening. Asian Pac J Cancer Prev, 16, 6177-86.

Mousavi SM, Montazeri A, Mohaghaghi A, et al (2007). Breast cancer in Iran: An Epidemiological Review, 13, 383-91.

Munsell MF, Sprague BL, Berry DA, et al (2014). Body mass index and breast cancer risk according to postmenopausal estrogen-progestin use and hormone receptor status. Epidemiol Rev, 36, 114-36.

Scheibel M,Bucciatelli E (2003). Breast cancer in young women: Clinico-pathological features and biological specificity. Breast, 12, 247-50.

Shakouri Prtovi P,Nami F (2004). Evolution of the mammographic findings in patients over 40 years of age with mammary disorders. Archive of SID, 9, 72-82.

Shannon C, Smith IE (2003). Breast cancer in adolescent and young women. Eur J Cancer, 39, 2632-42.

Sickles EA (2007). Wolfe mammographic parenchymal patterns and breast cancer risk. AJR, 188, 301-3.

Taheri NS, Bakhshandeh nosrat S, Tabiei MN (2012). Epidemiological pattern of breast cancer in Iranian women: is there an ethnic disparity? Asian Pac J Cancer Prev, 13, 4517-20.

Zulfiqar MA, Rohazly I, Rahmah MA (2011). Do the majority of Malaysian women have dense breasts on mammogram? Biomed Imaging Interv $J, 7,14$ 